\title{
The influence of channel models on simulated MIMO performance
}

\author{
Markus Herdin, Gerhard Gritsch, Biljana Badic and Ernst Bonek \\ \{markus.herdin, gerhard.gritsch, biljana.badic, ernst.bonek\}@nt.tuwien.ac.at \\ Institut für Nachrichtentechnik und Hochfrequenztechnik \\ Technische Universität Wien \\ Gußhausstrasse 25/389, A-1040 Vienna, Austria
}

\begin{abstract}
This paper shows that the simulated performance of MIMO systems is strongly influenced by the choice of the channel model. We investigated the performance of uncoded $4 \times 4$ and $6 \times 8$ MIMO systems without channel knowledge at the TX, when using either measurement data directly, the well-known 'Kronecker' model or a novel channel model that eliminates some of the deficiencies of the 'Kronecker' model. We found up to $5 \mathrm{~dB}$ difference in the required SNR for a specific target BER for the different channel models considered. The popular 'Kronecker' model, apart from predicting the mutual information inaccurately, also renders the BER erroneously when compared to the measurement, whereas the novel channel model performs better in both quantities.
\end{abstract}

\section{INTRODUCTION}

The performance of future multiple-input multiple-output (MIMO) systems is strongly influenced by the properties of the underlying radio channel. Modeling the radio channel accurately is essential for system design and performance estimation. Inaccurate channel models can lead to sub-optimum transmission schemes and wrongly estimated performance of chosen algorithms.

A very popular MIMO channel model is the so called 'Kronecker' model (e.g. [1] [2] [3]), which assumes separable transmitter and receiver correlation. If this assumption is valid, separate optimization at transmit and receive side is feasible. Kai Yu et. al [4], has shown that for systems with less than three transmit and receive antennas, aggregate statistics like average capacity can well be predicted by this model. However, this model shows remarkable deficiencies when compared to measurements with large arrays (i.e. more than 4 antennas at both link ends) (e.g. [5] and [6]). It leads to wrongly rendered multipath structure and significantly reduced MIMO capacity compared to the underlying measurement data. To overcome these problems, a new channel model was proposed by Weichselberger et al. [7] that models the MIMO channel in a more general way and includes the 'Kronecker' model as a special case.

A mismatch in capacity and multipath structure is clearly an indicator for wrong modeling but is this relevant for the performance of a 'real' system? How large are differences in, say, BER or required SNR for a target BER when using different channel models in MIMO system simulations? To answer these questions we will analyze the performance of a MIMO system with uncoded QPSK transmission and minimum mean square error (MMSE) detection in the receiver.

\section{SyStem MOdEL AND TRANSMission SCHEMES}

In this investigation we consider a transmission scheme without channel knowledge at the transmitter with the system model

$$
\mathbf{y}=\mathbf{H s}+\mathbf{n}
$$

Here, $\mathbf{y}$ denotes the receive vector, $\mathbf{H}$ the MIMO channel matrix, $\mathbf{s}$ the transmit vector with correlation $\mathbf{R}_{\mathrm{s}}=$ $\mathrm{E}\left\{\mathbf{s s}^{H}\right\}=P_{t} / n_{t} \cdot \mathbf{I}_{n_{t}}$, i.e. equal transmit power $P_{t} / n_{t}$ for each transmit antenna. The received noise vector $\mathbf{n}$ is assumed to be uncorrelated, hence $\mathbf{R}_{\mathrm{n}}=\mathrm{E}\left\{\mathbf{n n}^{H}\right\}=\sigma_{n}^{2} \cdot \mathbf{I}_{\mathrm{n}_{\mathrm{r}}}$. $\mathbf{I}_{n}$ denotes the $n \times n$ identity matrix, and $n_{t}$ and $n_{r}$ are the number of transmit and receive antennas. The channel matrices are normalized such that

$$
\mathrm{E}\left\{\sum_{i=1}^{n_{t}} \sum_{j=1}^{n_{r}}\left|h_{i j}\right|^{2}\right\}=n_{r} n_{t}
$$

which means that the average SISO pathloss is normalized to unity, and the average receive SNR at the receive antennas is given by

$$
\rho=\frac{P_{t}}{\sigma_{n}^{2}} .
$$

We will consider a $4 \times 4$ system with 4 independent data streams and a $6 \times 8$ system with 6 independent data streams. At the receive side, a minimum mean square error (MMSE) receiver is used, followed by a separate detection for each data stream.

\section{MIMO CHANNEL MODELS}

We compare the 'Kronecker' and the already mentioned novel channel model in this paper. In the following, we will refer to the latter as Weichselberger model for the original inventor of this channel model. 


\section{A. The 'Kronecker' MIMO Channel Model}

The basic assumption of the 'Kronecker' model is that the full channel correlation matrix can be modeled by the Kronecker product of the transmit and receive correlation matrix [3]. This leads to the stochastic channel model

$$
\tilde{\mathbf{H}}=\frac{1}{\sqrt{\operatorname{tr}\left\{\mathbf{R}_{\mathrm{Rx}}\right\}}} \mathbf{R}_{\mathrm{Rx}}^{1 / 2} \mathbf{G}\left(\mathbf{R}_{\mathrm{Tx}}^{1 / 2}\right)^{\mathrm{T}} .
$$

Here, $\mathbf{R}_{\mathrm{Tx}}$ is the transmit and $\mathbf{R}_{\mathrm{Rx}}$ the receive correlation matrix, $\mathbf{G}$ a random matrix with independently identical distributed complex Gaussian entries, $(\cdot)^{1 / 2}$ denotes the matrix square root, $\operatorname{tr}\{\cdot\}$ the trace operator and $\tilde{\mathbf{H}}$ is the resulting channel matrix realization. The main deficiencies of this model are that the multipath structure is not rendered correctly, and, as result of this, the estimated mutual information of this channel model is typically below that one estimated directly from the measurement data [6].

\section{B. The Weichselberger MIMO Channel Model}

The Weichselberger model was presented in [7]. There it was also shown that it is able to reproduce the multipath structure and the mutual information significantly better than the 'Kronecker' model. Actually, the 'Kronecker' model is a special case of this channel model. Channel matrices are generated by

$$
\tilde{\mathbf{H}}=\mathbf{U}_{\mathrm{Rx}}(\tilde{\mathbf{\Omega}} \odot \mathbf{G}) \mathbf{U}_{\mathrm{Tx}}^{T}
$$

where $\mathbf{U}_{\mathrm{Rx}}$ and $\mathbf{U}_{\mathrm{Tx}}$ are the receive and transmit Eigenvector matrices given by the Eigenvalue decomposition of $\mathbf{R}_{\mathrm{Rx}}$ and $\mathbf{R}_{\mathrm{Tx}}$, respectively, $\mathbf{G}$ is a random matrix with independently identical distributed complex Gaussian entries and $\tilde{\Omega}$ is the element-wise square root of the power coupling matrix $\boldsymbol{\Omega}$. The power coupling matrix describes the coupling from all transmit Eigenmodes to all receive Eigenmodes. Based on measurements it is simply estimated using the estimated transmit and receive Eigenbasis:

$$
\boldsymbol{\Omega}=\mathrm{E}\left\{\left(\mathbf{U}_{\mathrm{Rx}}^{H} \mathbf{H U}_{\mathrm{Tx}}^{*}\right) \odot\left(\mathbf{U}_{\mathrm{Rx}}^{T} \mathbf{H}^{*} \mathbf{U}_{\mathrm{Tx}}\right)\right\} .
$$

\section{Measurement Setup and Scenario}

The measurements used for evaluating the performance of the considered MIMO systems were taken at the Institute of Communications and Radio-Frequency Engineering, Vienna University of Technology. A detailed description of the measurements can be found in [8]. The measurements were performed with the RUSK ATM wideband vector channel sounder [9] with a measurement bandwidth of $120 \mathrm{MHz}$ at a center frequency of $5.2 \mathrm{GHz}$. At transmit (TX) side, we used a $\lambda / 2$ spaced virtual $20 \times 10$ matrix formed by a horizontally omnidirectional antenna and at receive (RX) side an 8element uniform linear array (ULA) of printed dipoles with $0.4 \lambda$ inter-element spacing and $120^{\circ} 3 \mathrm{~dB}$ beamwidth.
We measured 193 frequency samples of the transfer function between all TX and RX elements with a fixed transmitter at 24 different RX positions. For each position the RX antenna was rotated to three $120^{\circ}$ angularly spaced directions giving in total 72 different measurement scenarios. By grouping 8 adjacent virtual TX antennas within the virtual $20 \times 10 \mathrm{TX}$ matrix together, we formed a virtual 8-element TX array. This 8-element ULA was moved virtually over the TX matrix to create 130 different realizations of the MIMO channel matrix. We used both the spatial and frequency realizations as realizations of the MIMO channel, which gives therefore in total $130 \cdot 193=25090$ realizations of an $8 \times 8$ MIMO channel matrix. These realizations were used to simulate the performance of the considered MIMO systems and also to estimate the parameters for the considered channel models.

\section{Evaluation}

\section{A. Channel Matrix Realizations}

To evaluate the performance of the considered MIMO systems, we used all available (25090) channel matrix realizations from the measurement and created the same number of channel realizations based on the two different MIMO models. The necessary parameters (the transmit and receive correlation matrices) were extracted from the measurements by

$$
\begin{aligned}
& \mathbf{R}_{\mathrm{Tx}}=E\left\{\mathbf{H}^{T} \mathbf{H}^{*}\right\}=\mathbf{U}_{\mathrm{TX}} \boldsymbol{\Lambda}_{\mathrm{TX}} \mathbf{U}_{\mathrm{TX}}^{H} \\
& \mathbf{R}_{\mathrm{Rx}}=E\left\{\mathbf{H} \mathbf{H}^{H}\right\}=\mathbf{U}_{\mathrm{RX}} \boldsymbol{\Lambda}_{\mathrm{RX}} \mathbf{U}_{\mathrm{RX}}^{H}
\end{aligned}
$$

where the expectation was performed over the given channel realizations. For the 'Kronecker' model, the correlation matrices $\mathbf{R}_{\mathrm{TX}}$ and $\mathbf{R}_{\mathrm{RX}}$ and for the novel channel model the corresponding Eigenbasis $\mathbf{U}_{\mathrm{TX}}$ and $\mathbf{U}_{\mathrm{RX}}$ are needed.

For the $6 \times 8$ system we used the outer $6 \mathrm{TX}$ antennas (1-3 and 6-8) and all receive antennas and for the $4 \times 4$ system the first 4 transmit and receive antennas for evaluation and parameter extraction.

\section{B. Performance Measures}

We considered two different metrics to compare the two channel models with the measurements:

- The average mutual information for a fixed average receive SNR (20dB) when the channel is unknown at TX and

- the SNR at which a fixed target bit error rate $\left(10^{-2}\right.$ or $10^{-3}$ ) is reached.

The average mutual information in $b i t /(s \cdot H z)$ was calculated by

$$
C=\mathrm{E}\left\{\log _{2} \operatorname{det}\left(\mathbf{I}_{n_{r}}+\frac{\rho}{n_{t}} \cdot \mathbf{H} \mathbf{H}^{H}\right)\right\}
$$


with the expectation performed over the channel realizations. Here, $\rho$ the average receive SNR and $\mathbf{H}$ one MIMO channel matrix realization.

To calculate the SNR value for the target BER, we interpolated the logarithmic BER between the simulated SNR values in $\mathrm{dB}$. This method turned out to be accurate enough for this purpose.

\section{RESUlTS}

Figure 1 depicts the performance of the MMSE receiver for a selected measurement scenario. The BER for a $4 \times 4$ system (top) and a $6 \times 8$ system (bottom) is shown when either the 'Kronecker' model, the Weichselberger model or the measurement data itself was used for the simulation. The 'Kronecker' model leads to significantly overestimated BER, whereas the Weichselberger model underestimates the BER compared to the measurement but shows a substantial better fit. We clearly see that the choice of the channel model significantly influences the simulated BER results. At the target BER of $10^{-3}$ the deviation is up to $5 \mathrm{~dB}$ for the $6 \times 8$ system.
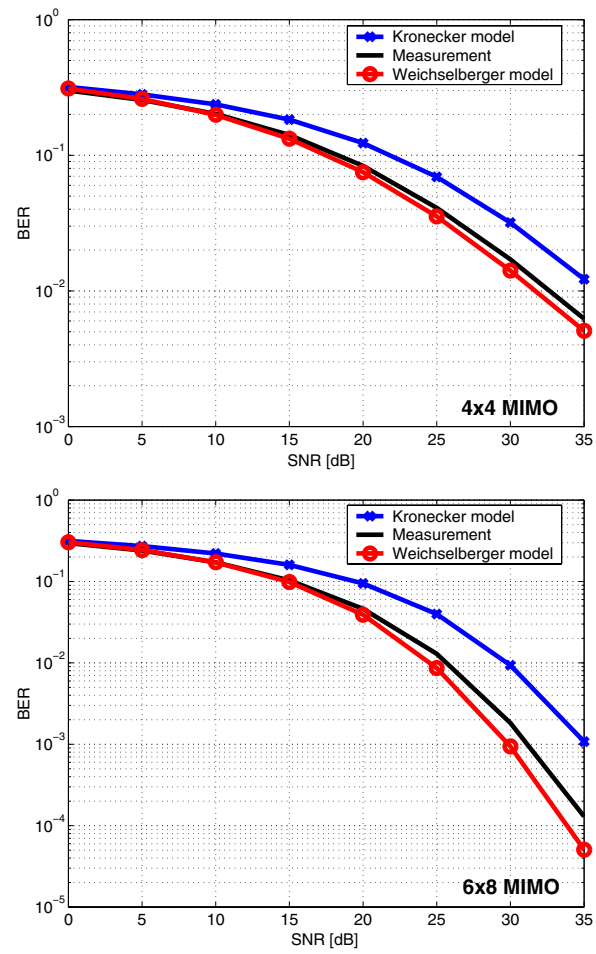

Fig. 1. Performance of an MMSE detector for a $4 \times 4$ MIMO system (top) and a $6 \times 8$ MIMO system (bottom) for measurement, 'Kronecker' and Weichselberger model, one selected scenario

One scenario, of course, is not sufficient to draw general conclusions. Therefore, we consider in the following all available scenarios, but focus only on the SNR that is required to reach the target BER. As a consequence of the different steepness of the BER/SNR curves we chose a target BER of $10^{-2}$ for the $4 \times 4$ MIMO system and $10^{-3}$ for the $6 \times 8$ MIMO system.
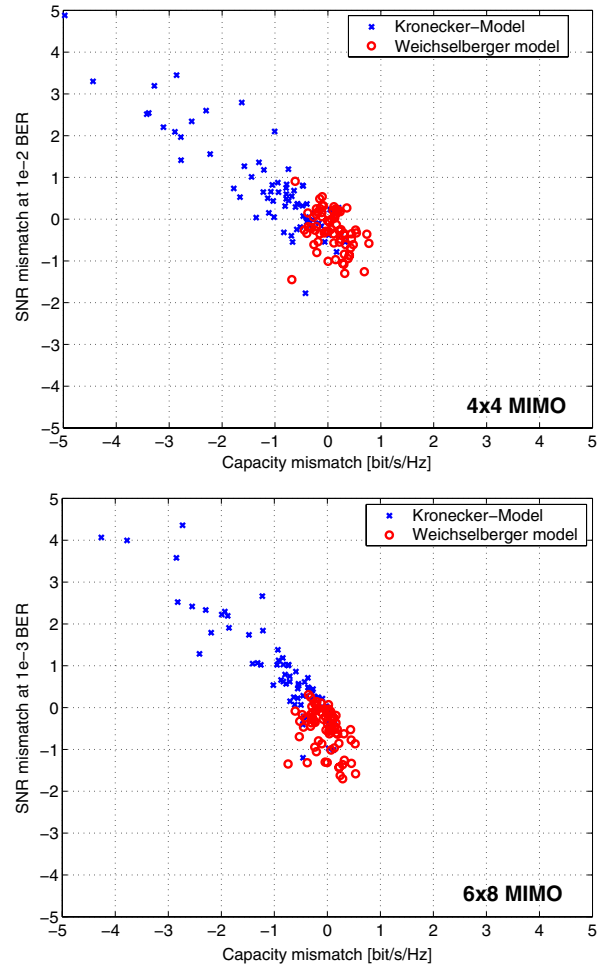

Fig. 2. SNR mismatch of BER performance with MMSE detector vs mutual information mismatch for Kronecker and Weichselberger model compared with the measurement data itself, top: $4 \times 4$ MIMO system, bottom: $6 \times 8$ MIMO system; for all 72 scenarios

As described in the evaluation section, this target SNR was interpolated between the simulated SNR values. Additionally, we calculated the average mutual information, usually called 'mean capacity', that can be reached without TX channel knowledge assuming an average receive SNR of $20 \mathrm{~dB}$.

To compare these results with the measurement, which we consider as the "true' MIMO channel, we define two metrics that quantify the fit of the channel model with the measured data:

- The SNR mismatch, the difference between the SNR value at the target BER when using the channel model and the SNR value at the target BER when using the measurement data itself, and

- the mutual information mismatch, the difference between the mutual information for the channel model and the mutual information for the measurement data.

Whereas, for a perfect channel model, both metrics should be zero, the results for the 'Kronecker' and the Weichselberger model show that they are not (Figure 2).

Each blue cross corresponds to one measurement scenario when using the 'Kronecker' model and each red circle when using the Weichselberger model. On the x-axis the mutual information mismatch and on the y-axis the SNR mismatch is shown.

The mutual information mismatch for the 'Kronecker' model 

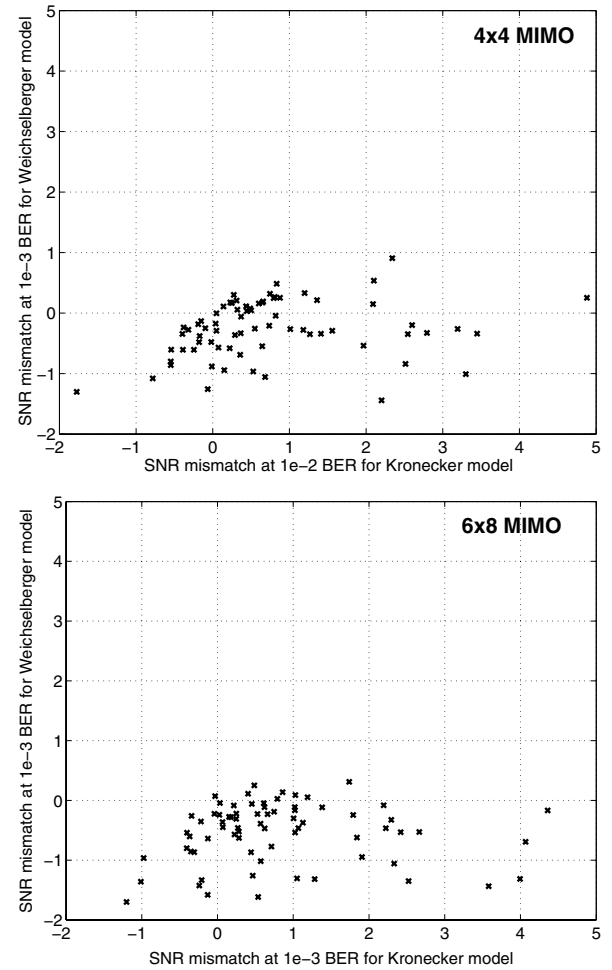

Fig. 3. SNR mismatch of Weichselberger model vs 'Kronecker' model, top: $4 \times 4$ MIMO system, bottom: 6x8 MIMO system, for all 72 scenarios

is much larger than that for the novel channel model [6]. Also, it can be seen that the 'Kronecker' model typically underestimates the mutual information, whereas the novel channel model slightly overestimates it. However, not only the mutual information is significantly worse predicted by the 'Kronecker' model but also the BER or, equivalently, the SNR value at which the considered target BER is reached. The 'Kronecker' model overestimates the SNR at target BER by up to $5 \mathrm{~dB}$, whereas the underestimation of the novel channel model is always below $2 \mathrm{~dB}$.

The important conclusion here is that the mutual information mismatch, which was discovered for the Kronecker model [6], shows also up as an SNR mismatch at a specific BER level. This means that, although mutual information is a rather global measure for a MIMO channel, a mismatch in this quantity is indicative of erroneous BER results as well. More important, the influence of the chosen channel model becomes evident again.

Another question we want to answer is whether the SNR mismatch of the 'Kronecker' model is related to the SNR mismatch of the novel channel model or not. To this end Figure 3 shows the SNR mismatch of the novel channel model vs the SNR mismatch of the 'Kronecker' model for each scenario.
The clear answer is no. The errors in the two channel models do not occur in a correlated manner. The novel channel model performs better on average but not necessarily for each scenario.

\section{SUMMARY AND CONCLUSION}

We investigated the performance of uncoded $4 \times 4$ and $6 \times 8$ MIMO systems without channel knowledge at the TX, when using either measurement data directly, the well-known 'Kronecker' model or a novel channel model that eliminates some of the deficiencies of the 'Kronecker' model. The choice of the channel model turns out to be critical for predicting BER and required SNR to reach a specific target BER. The channel models considered showed up to $5 \mathrm{~dB}$ difference in the SNR at the target BER. The popular 'Kronecker' model, apart from predicting the mutual information inaccurately, also renders the BER vs SNR curves erroneously when compared to the measurement, whereas the novel channel model is able to model both quantities significantly better.

\section{ACKNOWLEDGMENTS}

We would like to thank Helmut Hofstetter (Forschungszentrum Telekommunikation Wien, ftw.) for help with the measurements and T-Systems Nova $\mathrm{GmbH}$ for providing an eight element uniform linear array of printed dipoles.

\section{REFERENCES}

[1] C.-N. Chuah, J. M. Kahn, and D. Tse, "Capacity of multi-antenna array systems in indoor wireless environment," IEEE Global Telecommunications Conference, vol. 4, pp. 1894-1899, 1998.

[2] K. Pedersen, J. Andersen, J. Kermoal, and P. Mogensen, "A stochastic multiple-input-multiple-output radio channel model for evaluation of space-time coding algorithms," IEEE VTC Fall, vol. 2, pp. 893-897, September 2000.

[3] J. Kermoal, L. Schumacher, K. Pedersen, P. Mogensen, and F. Frederiksen, "A stochastic MIMO radio channel model with experimental validation," IEEE Journal on Selected Areas in Communications, vol. 20, pp. 1211-1226, Aug. 2002.

[4] K. Yu, M. Bentsson, B. Ottersten, and D. McNamara, "Second order statistics of NLOS indoor MIMO channels based on $5.2 \mathrm{GHz}$ measurements," Globecom, vol. 1, pp. 156-160, Nov 2001.

[5] H. Özcelik, M. Herdin, H. Hofstetter, and E. Bonek, "A comparison of measured $8 \times 8$ MIMO systems with a popular stochastic channel model at 5.2 GHz," ICT, vol. 2, pp. 1542-1546, 2003.

[6] E. Bonek, H. Özcelik, M. Herdin, W. Weichselberger, and J. Wallace, "Deficiencies of a popular stochastic MIMO radio channel model," International Symposium on Wireless Personal Multimedia Communications, WPMC, Yokosuka, Japan, October 2003.

[7] W. Weichselberger, H. Özcelik, M. Herdin, and E. Bonek, "A novel stochastic MIMO channel model and its physical interpretation," International Symposium on Wireless Personal Multimedia Communications, WPMC, Yokosuka, Japan, October 2003.

[8] H. Özcelik, M. Herdin, R. Prestros, and E. Bonek, "How MIMO capacity is linked with multipath distribution," International Conference on Electromagnetics in Advanced Applications, Torino, Italy, 2003, pp. 775-778, September 2003.

[9] R. Thomä, D. Hampicke, A. Richter, G. Sommerkorn, A. Schneider, U. Trautwein, and W. Wirnitzer, "Identification of time-variant directional mobile radio channels," IEEE Transactions on Instrumentation and Measurement, vol. 49, pp. 357-364, April 2000. 